\title{
Emojis as a new Visual Language in Advertising Creativity
}

\author{
Dr.Amira Kadry Yassin \\ Associate Professor, Art\&Design Academy, The Higher institute of Applied Arts, Graphics \& Advertis- \\ ing Arts dept. 6th of October City, Egypt
}

\begin{tabular}{l|l|l|l} 
Submit Date: 2020-05-10 16:13:18 & Revise Date: 2021-01-06 07:05:24 & Accept Date: 2021-01-07 22:53:48
\end{tabular}

DOI:10.21608/jdsaa.2021.29904.1037

Keywords:

Emoji, Emoji Marketing, Generation Z, Emojipedia

\begin{abstract}
:
Creativity, that magical force, is the foundation upon which the advertising business is built. Whenever it comes to Advertising "Creativity" is the critical and most important aspect to be looked upon. Advertisers all over the world are constantly trying to find the best way to grab the attention to appeal to the largest audience possible. They are constantly looking for eye-catching innovative visual elements to implement in their designs. As the Advertising world is undergoing a great period of change; visual communication has come a long way from cave paintings to tweets, emails to hashtags. If we take a look back into our ancient history as a civilization; we will see how strongly it relied on illustrations to communicate. Naturally, humans are visual creatures who thrive on the ability to use images to convey their emotions. Modern communication technology has created Emojis, a brand new symbolic language that became an important part of interpersonal communication. It's surprising how quickly Emojis became mainstream. And now, they are being used in advertising messages especially targeted to Generation which is mobile-focused and their primary way of communicating is through mobile that has thousands of Emojis. Whether they are texting, tweeting, snapping or anything else, they live on their phones. Emojis gave them an easier way to express ideas, and their popularity continues to grow. People's enthusiasm for Emoji is not just about chasing new trends, but also a deep functional appeal for communication. Customers are now adapted to the constantly evolving language in everyday lives, Emojis are now found in every corner of the digital world, proving that "a picture is worth a thousand words." In this new era; advertisers are not tracking customers' Google keywords or their gender and location anymore. Advertisers are developing ads to target them based on the Emojis they use. Social media was in fact the catalyst for the immense popularity of Emojis, they provided advertisers with a welcome opportunity to interact and connect with a new generation of consumers that expect more meaningful and creative engagement, for advertisers learning to work with Emojis has been an essential part of adapting to changing market expectations.
\end{abstract}


This paper aims to look closely and explore Emojis as the world's fastest-growing form of visual communication, mainly as a new visual Advertising language in the digital information age, and how it contributes in engaging \& persuasion of target audience especially Generation Z.As it is an important to speak the language of your target audience. The paper will present a brief history of Emojis, study Emoji's various characteristics and how could they be utilized successfully in Advertising creativity through looking closely on selected International Emoji inspired Ads. Finding that Emojis are the New advertising Age visual language, They are effective visual tools in the new era of advertising communication when used upon previous study of the target audience with respect to cultural differences .Emojis aren't just a trend they will stay for a while as an inspirational tool for designers. They are the next evolution of communication.

\section{Introduction}

Pictures speak a thousand words. Especially if those pictures are funny graphic icons. Emojis those new small digital icons that are used to express ideas, emotions in chat messages all over the social media; smiley face, thumbs up and so many more in today's electronic communication. Emojis have gone far from being virtually unknown to being the central topic in internet communication. They became a significant development in contemporary communication, deserving serious attention for their expected positive impact on the Advertising industry. Many advertisers are now integrating Emojis into their campaigns, as a way to better tailor their messages and to reach more target audiences. For years, advertisers have been chasing the "Millennial" market for their big size, influence, growing incomes and their big appetite for digital media. But now, the eldest Millennials are approaching their 40s and advertisers are shifting their attention to Generation Z ; those who were born after the turn of the century, who grew up in the social, mobile digitally-connected world. They communicate with text messages, increasingly with Emojis . This new language has become so important that there are now Emoji translators; EMOJIPEDIA is the most wellknown website to look for Emojis meanings. They took over the marketing world, with an array of brands using them to communicate with their audiences on social media on a regular basis, smoothly penetrating into their customers' everyday conversations by using the language they speak. Those icons that emerged as a compensatory universal language. (Danesi, 2016, p. 5). Advertisers are taking the advantage of this ginormous spread of this digital symbolic language and are keen to implement it creatively in their advertising messages.

\section{Research Objective}

To explore how Emojis are a new Advertising universal visual language and how does it contribute in achieving a strong visual impact in creative adverts.

\section{Research problem}

The research problem can be formulated in the following questions:

- What Are Emojis?

- Could Emojis be used as a universal advertising language?

- Are Emojis an effective visual tool in advertising?

- How can you employ Emojis successfully in advertising creativity especially adverts for Generation $\mathrm{z}$ ?

\section{Hypothesis}

- The use of Emojis as a new Visual design element; may lead to a more effective way of visual communication in Advertising, as the clarity of their form makes the advert more familiar, understandable, direct and powerful and may lead to advert effectiveness.

- The use of Emojis increases audience engagement to the advert, as consumers are more likely to react positively to familiar images.

- Emojis enriches communication and makes it more enjoyable.

- Using Emojis help improve communication with your audience.

\section{Methodology}

The study is based on the previous literature, it follows the Descriptive method to explore how Emojis became the new Advertising universal visual language; and how does it contribute in achieving a strong visual impact in creative ads. Followed by looking closely $\&$ analytically through international creative Emoji inspired campaigns.

\section{Theoretical Framework}

Emojis Defined

- The word "Emoji" is an English adaptation of a Japanese word .The E (絵) means picture and the moji (文字) stands for letter or character. Emoji means a "picture word". (Springer, 2009, p. 7) 
- It is a small graphic image depicting an array of human emotions. Usually resembling a yellow face with a pair of black eyes and a mouth, they can be found on nearly every smartphone across the globe. Emojis follow a coding standard called Unicode, which means that no matter what device you are using, you'll most likely be able to read an Emoji the way it was intended. (Lucas, 2016, p. 17)

- The word Emoji, was added to the Oxford Dictionaries in 2013, and eventually crowned the Top Word of 2014 by the Global Language Monitor. This marked the first time a symbol was chosen. The language is even celebrated every July 17th on World Emoji Day. (Seargeant, 2019, p. 17)

A Brief History of Emojis

Before the era of Emojis, we had "Emoticons". Emoticons are smiley faces, frowning faces and other creative compositions created with symbols available on your keyboard. They have been used for years on the internet via messaging and emails. It all started with Emoticons; those facial expressions made out of punctuation marks which made their first appearance in an issue of a magazine back in the year 1881. It was just about 100 years later, in 1982 that Emoticons were first incorporated into computer language, An Emoticon is a text-based smiley face, and then they became more mainstream after the turn of the millennium. Whereas an Emoji is an image that depicts an expression, and is standardized across several platforms: iOS, Windows, OS X, and Android. The modern Emojis we know today, only became popular around the year 2011.

While Emojis are animated digital images, Emoticons are emotions and facial representations communicated through text. Unlike Emojis, Emoticons can be created using characters found on a keyboard. (Benenson, How to speak Emojis, 2016, p.

\section{6)}

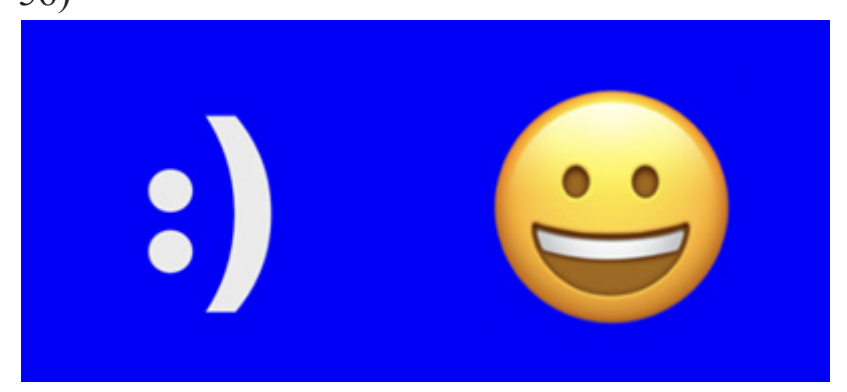

Fig (1) Emoticon \& Emoji

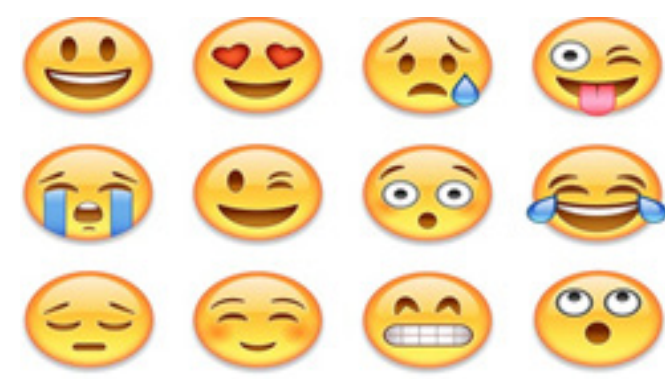

Fig (2) Emojis

The most popular Emoji's on APPLE \& ANDROID platforms are:

- Face with tears of joy

- Red Heart

- Loudly crying face

- Smiling face with heart eyes

- Face blowing a kiss

- Face with rolling eyes

- Smiling face with smiling eyes

\section{Emoji marketing}

Is the practice of creating marketing campaigns which involve the use of Emojis. These include social media posts with Emoji used along with words, used as hash tags, used in graphics, Emoji merchandise, Emoji domains and any other creative way that a marketer or designer can think of to use Emoji. (Eskilson, 2019 - 3rd Edition, p. 115) Among the most famous examples of Emoji Marketing; is the Facebook Reactions. One of the largest Emoji campaigns of the last couple of years is the introduction of the Facebook reactions Emoji. It might not seem like a marketing campaign at first, but if you think about it, everything about Facebook is marketing and business. Their Facebook reaction Emoji are most definitely a marketing campaign, one that we have accepted as a daily and normal part of our, liking, smiling and, well, sad reacting. It is the most successful of all campaigns to date. It also paved the way into a new era of Emoji marketing

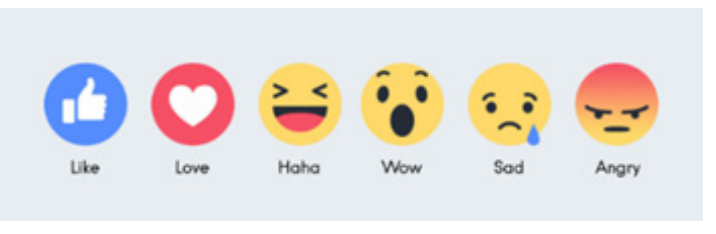

Fig (3) Facebook reactions

\section{Emojis in Advertising}

It is the use of Emojis in marketing \& advertising messaging strategies, to improve engagement rates, as the use of Emojis can go far beyond just a simple icon. 
Several prominent brands have already turned Emojis into designs that captured their audience's attention for many reasons:

\section{Emojis Scale Past Language Barriers}

it's not a secret that Emojis are universally understood by people everywhere in the world now. So, if a brand is interested in expanding into a new state or country, using Emojis as part of the initial marketing strategy could be a great way to gain the attention of the targeted customers.

\section{Emojis improve the Rate of Engagement}

Text can quickly be boring. And given the volume of wordy advertising campaigns being out every day, using Emojis can be a refreshing change for the end user. In fact, using Emojis can improve engagement to advertising by nearly $48 \%$ on platforms like Instagram.

\section{Emojis are easily processed by the Human Brain} The human brain processes visuals several times faster than text. Scientists have found that we can process entire images in as few as 13 Milliseconds. Brands can use this to their advantage and convey a lot more with a single Emoji than they could with a string of words.

\section{Emojis offer Opportunities for Innovation}

Unlike text, which can only be manipulated to a certain extent, Emojis offer larger scope for creativity and innovation. There are around 3000 Emojis in use today, and brands can even create their own Emojis to convey their unique message to the consumers. The possibilities are endless when using Emojis.

\section{Emojis outstanding Visual power}

Emojis are visually memorable. Scientists discovered that when we look at a smiley face online, the same parts of the brain are activated as if we looked at a real human face.

\section{Simplicity}

Emojis are quick and easy to use and adapt to the advertising message.

\section{Emotional impact:}

Emojis convey emotions more than "text only" messages. They tend to trigger the emotional part of our brains, as well as they enhance any messages that we send out.

\section{Broad and targeted reach:}

Just about everyone uses them nowadays, but particularly the coveted GenerationZ target market. (Danesi, 2016, pp. 85-90)

How to Successfully Use Emojis in Advertising Campaigns

\section{Make Them Relevant}

Emojis are great when used correctly. The first step in making sure that they're appropriate is to ask yourself if the audience uses them, and if they fit in well with the brand itself. Bearing in mind that not all content needs an Emoji.

\section{Look to Bios/Posts for Emoji Tips}

When targeting a specific demographic on social platforms, learning the voice that appeals to that demographic is important. So if you are looking to do some quick research, skip straight to the bios and maybe a few recent posts. Studying the Emojis they use in their bios will give you ideas for ways to comfortably approach your target audience.

\section{Leverage Social Intelligence}

Certain social intelligence tools allow brands to dissect a target audience to determine their most commonly used Emojis. Since advertising campaigns usually focus on a particular customer, these tools will allow brands to ascertain Emojis at a cluster or group level. Brands can then use this intelligence to properly employ them in advertising campaigns.

\section{Consider your brand personality first}

Always ask yourself if Emojis are appropriate to use with your brand or not, if your company works in fashion, sports, music, or any other form of entertainment, it's very likely that Emojis are appropriate for your brand. However, if you're offering a more serious service, there's a strong chance of missing your mark.

\section{Don't overuse Emoji's}

It is a popular method of communicating with audiences nowadays, particularly the younger generations, but an overuse might seem patronizing and could cause Emoji fatigue.

\section{Make sure you know what they mean}

Don't use an Emoji in a design, if you don't know what it means, or if you think its meaning would be confusing. If you're unsure whether an Emoji has an alternate 
meaning, you can look it up on Emojipedia first.

\section{Don't let Emoji's confuse your message}

While Emojis can add fun being an engaging element to your message, they're not a replacement for the message itself. Don't replace important words with Emojis, since more often than not they'll just obscure what you're trying to say. (Long, Tips to Using Emojis in Your Marketing Campaigns, 2017)

\section{Emojis tactics in Advertising Creativity}

Emojis are easy to create, easy to implement in the right design and strategy, they can steal attention and communicate value propositions in a blink. Here are a few examples of effectively reaching target audiences through Emoji Inspired Adverts.

\section{Intrusion}

Is a technique that uses packaging items as mediums for advertisements using the value of a medium which equates to using the role of the medium, as a way of connecting the medium to the consumers. (Sherin, 2013, p. 125)

\section{Pepsi}

Pepsi, used Emojis; the wordless icons as the centerpiece of a unique advertising campaign titled "Say it with Pepsi". Where Pepsi used more than 70 global and locally uniquely designed Emojis printed on cans, bottles and cups all over the world, called it the PepsiMoji language sparking unexpected conversations and action around the world through its customers using Emojis. This campaign was mainly created upon the fact that Millennials and Generation $\mathrm{Z}$ believe voicemail takes too long, email requires too much effort, while texting is simple, fast, and direct. Emojis are considered to be the next evolution of communication. They can transcend through language barriers. Pepsi wanted People to use their drink as a way of self-expression. Pepsi took this Emoji based creative approach to encourage consumers to spark emotive, unexpected conversations around the world, as Emojis, represent a range of unlimited emotions without saying a word.

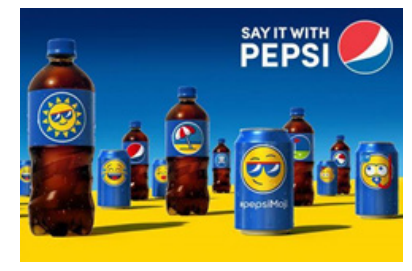

Fig (4)

\section{2- Transformation}

Is a technique that uses changes and alterations to physical states of physical materials to present advertising contents. These changes include changes in size, color, shapes, content or story and locations, changes through addition and removals and changes through replacements. (Stoklossa, 2007, p. 127)

\section{Dangers of online chatting with children campaign} This French campaign used Emojis in a very creative way to create brilliant visuals to warn parents against the dangers their children might face while chatting with strangers online and to Protect Children from Online Sexual Abuse. Through creating a real-life Emoji doing similar facial expressions, the idea behind using real life Emojis, was to show the disturbing reality that could lie behind the fun Emoji face in a child's chat box. As the tagline on the adverts question\& warn: "who's really chatting online with your child?" When a predator makes contact with a child online, they will first try to gain the child's trust. They might even try and pass themselves off as another child, and they will do this by speaking the same online language we all use on daily basis.
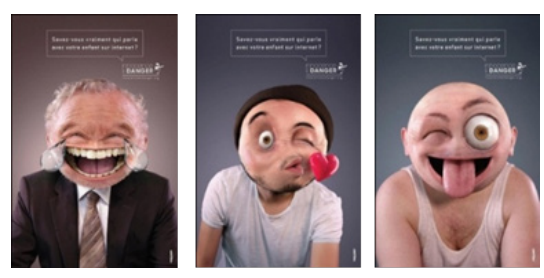

Fig (5)

\section{3- Installation}

Is a technique that uses creations of new items to serve as new mediums of marketing and advertising presentations. "Don't text and Drive" An Ambient Advert of a Driving Awareness Emoji inspired campaign. The main idea was turning a wrecked Car into a Mangled Emoji for expressing the main Tag line of the campaign. 'Don't Text and Drive' ;using popular Emoji's that sticks its tongue out and the one with a damaged tire in place of the cool sunglasses .

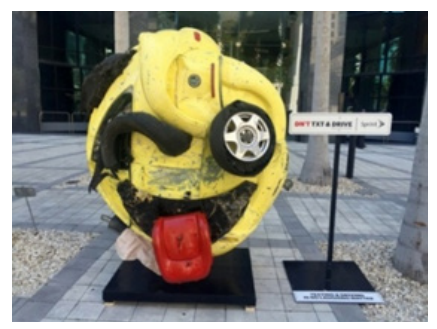

Fig (6) 


\section{4- Illusion}

Is a technique that focuses on distortions of the target groups' perceptions of reality and results in them being unable to distinguish fact from fiction. These can be done through mimicking of existing items, usages of 3D technology in presentations, creation of contents and storyboards and creating misunderstandings and misperceptions. (Eskilson, 2019 - 3rd Edition, p. 256) Ford Don't Emoji and Drive

Ford builds the shape of an old manwalking with a stick using different colors and sizes of Emojis, to demonstrate that writing messages while driving ends up that you may not see who crosses the road.

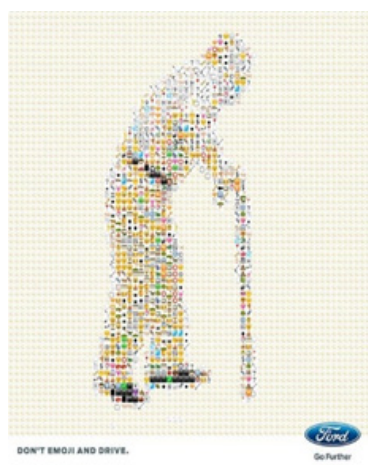

Fig (7)

\section{Vodafone}

To express the idea of outstanding internet speed in $4 \mathrm{G}$ Vodafone services; Speed Tears was an Emoji inspired campaign, giving the illusion of fast speed through the eye-watering caused by attaining a very high speed. Something even the Emojis you are using will experience when sent from your Vodafone mobile phone.
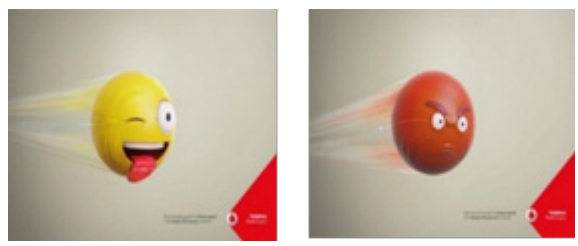

Fig (8)

\section{5- Metaphors}

This technique is useful to communicate complex ideas or Fig (9)

advertising messages. Metaphors help to communicate any idea quickly and vividly. A metaphor, uses two things - such as a vivid statement or a dramatic visual - to suggest another thing. Metaphors help to bring abstract ideas to life, to also represent unexpected risks in life through creative visuals. (Tellis, 2007, p. 256)

\section{Volkswagen}

As part of a text and drive campaign, Volkswagen featured a pretty bruised and battered Emoji with bandaged heads, knocked out teeth, smashed up sunglasses and bloodied face, showing the dangers of distracted driving Without having to use very many words at all, just Emojis.

At times, there is a combination of some of the 8 methods to ensure efficiency and success of the advertisements and marketing materials.

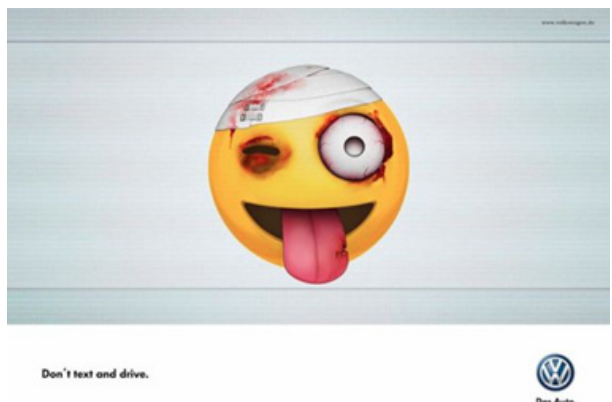

Fig (9)

\section{6- Exaggeration}

Exaggeration is an advertising technique that is both fun and effective. And it's easy to work with, easy to brainstorm. It's all about taking the basic idea you want to communicate, your concept, then exaggerate it. Take it to extremes. Push it beyond reason, beyond reality. In the copy, visuals, or both. Exaggerate a benefit, a problem, size, physical appearance. Exaggerate your exaggeration. Because exaggeration is interesting, powerful way to get communicate your concept. A small exaggeration is simply a misleading ad. (Eskilson, 2019 - 3rd Edition, p. 225)

\section{HONDA}

Honda launched the new model of its motorbike ,through an Emoji inspired campaign ,using exaggeration to express the adventures you could face while riding the motorbike in muddy roads. Exaggeration is used through a famous super excited Emoji with lots of mud and green leaves and tree branches stuck on it

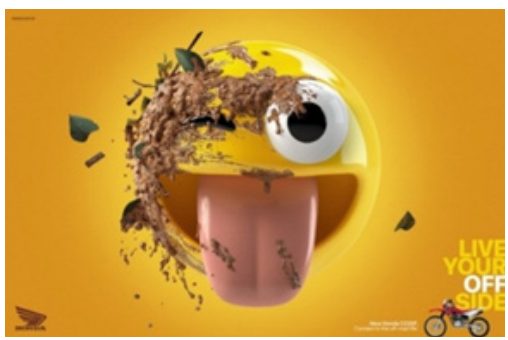




\section{7- Association}

Some advertising techniques rely mostly on psychology. Such is the case with the association technique, which is all about that the visuals in the graphic will create associations for the viewer. These associations can be feelings, ideas, places, or nostalgia. For association to be successful, a good bit of research must be done beforehand so that there is a deep knowledge of who the consumer is before deciding on what the association will be. (Velarde, 2017, p. 105)

\section{McDonald's}

The impressive thing about this marketing campaign is that McDonalds is using association in telling a story to the audience using only Emojis. This ad shows that an idea doesn't need to be complicated in order to be effective. And when it comes to simple, Emojis are tough to beat. The story says that if you are on a taxi heading to the Airport to catch your plane and dreaming of a fancy vacation,then heavy rain and lightning hits the road,so the flight is cancelled,you're angry taking the taxi back home then you think of a McDonalds meal to make it up for you and you're finally happy again . Because McDonald's is the first thing you think of to have a good time.

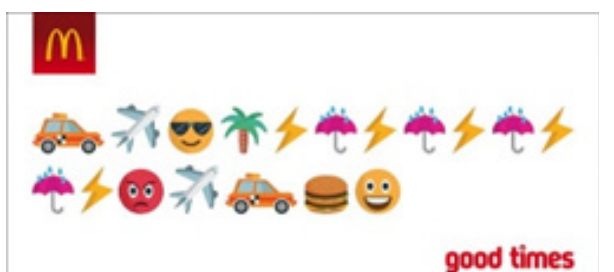

Fig (11)

Through the study of previous successful Emoji inspired advertising campaigns, it is obvious that Advertising Communication has taken on a new visual language, THE LANGUAGE OF TODAY;Emojis.

\section{Results}

- The use of Emojis as a new Visual design element; leads to a more effective way of visual communication in Advertising, as the clarity of their form makes the advert more familiar yet understandable, direct and powerful and leads to advert effectiveness.

- The use of Emojis increases audience engagement to the advert, as consumers react positively to familiar images\& make them easily recalled too.

- Emojis enriches communication and makes it more enjoyable.

- Emojis release marketing campaigns from stiff formal framework of marketing by making content lighthearted without compromising quality.

- Using Emoji can help improve communication with your audience.

- Emojis Scale Past Language Barriers, Emojis says what words couldn't say.

- Emojis should be used with caution and fine research when targeting different cultures and back grounds.

\section{It should be concluded that}

Emojis have become mainstream in both popular culture and Advertising. As advertisers continue to compete for younger audiences, we can expect to see the new language of Advertising continue to evolve .Bearing in mind that Emoji language is a dicey tool that brands need to think about twice before using as part of Advertising campaigns. They are effective but advertisers have to use Emojis sparsely, in the right places, and in the right manner. Use them clearly, without leaving a room for misinterpretation. Knowing one's audience and respecting them through the Emoji inspired Advertising message. Emojis are here to stay, and it's time to consider their newfound status as the VISUAL LANGUAGE of the digital age 


\section{References}

1. Benenson, F. (2016). How to speak Emojis. Andrews McMeel Publishing .

2. Danesi, M. (2016). The Semiotics of Emoji: The Rise of Visual Language in the Age of the Internet . USA: Bloomsbury Academic; Reprint edition.

3. Eskilson, S. J. (2019 - 3rd Edition). Graphic Design: A New History. Yale University Press.

4. Long, J. (2017). Tips to Using Emojis in Your Marketing Campaigns. Retrieved December 22, 2019, from Entrepreneur Middle East: https://www.entrepreneur.com/article/289704

5. Long, J. (2017, Feb). Tips to Using Emojis in Your Marketing Campaigns. Retrieved 12 22, 2019, from Entrepreneur Middle East : https:// www.entrepreneur.com/article/289704

6. Lucas, G. (2016). The Story Of Emoji. Prestel.

7. Seargeant, P. (2019). The Emoji Revolution: How Technology is Shaping the Future of Communication . UK: Cambridge University Press.

8. Sherin, A. (2013). Design Elements, Using Images to Create Graphic Impact. Rockport Publishers.

9. 9-Springer, P. (2009). Ads to Icons . KOGAN PAGE .

10. Stoklossa, U. (2007). Advertising: New Techniques for Visual Seduction. Thames \& Hudson.

11. Tellis, G. J. (2007). The SAGE Handbook of Advertising . SAGE Publications Ltd; 1st edition.

12. Velarde, O. (2017). Commonly Used Advertising Techniques in Visual Marketing. Retrieved 12 23, 2019, from visme.co: https://visme.co/ blog/visual-advertising-techniques/\#XAcjKwo0ApoBc8Gf.99 